\title{
Single persistently enlarged lymph node in the level ii region of the neck
}

\author{
Volume 7 Issue I - 2017 \\ Richard Law, Robin Lazar Miller , Saurin \\ Popat \\ Department of Otolaryngology University at Buffalo Jacobs \\ School of Medicine and Biomedical Sciences, USA \\ Correspondence: Richard Law Department of \\ Otolaryngology University at Buffalo Jacobs School of Medicine \\ and Biomedical Sciences Delawate Medical Group 1083 \\ Delaware Avenue Buffalo NY, USA, Tel 7I6-882-1023, \\ Email saurinpopat@gmail.com
}

Received: August 14, 2016 | Published: May 03, 2017 abuse. A complete head and neck exam was performed, and a $1 \times 1 \mathrm{~cm}$ rubbery, mobile nodule was palpated in the Level II nodal region. The remainder of the exam was negative and exam with FFL was also negative for any masses or lesions. She has a history of squamous cell carcinoma of her lower eyelid that was removed via wide local excision with clear margins. Other medical histories were noncontributory.

CT with contrast was negative for parotid or submandibular enlargement bilaterally and there was no evidence of parotid or cervical lymphadenopathy elsewhere. Ultrasonography of the mass revealed an ovoid solid structure measuring $1.5 \times 0.9 \mathrm{~cm}$ consistent with a lymph node with abnormal morphology. FNA was done on the mass. PET/CT showed increased uptake in the right upper neck, but no evidence of disease elsewhere in the body. An excisional biopsy of the mass with panendoscopy was performed. Multiple mucosal biopsies were taken during panendoscopy, and the frozen sections of these specimens were negative for malignancy. EBV and HPV testing were negative.

\section{What is the diagnosis?}

a. Metastatic squamous cell carcinoma: micrometastatic disease

b. Occult parotid malignancy with nodal involvement

c. Lymphoepithelial carcinoma

d. Benign inflammatory lymph node

\section{Diagnosis}

Lymphoepithelial Carcinoma. The final pathology of the mass was lymphoepithelial carcinoma with an unknown primary: TxN1M0. The patient was given the option of close monitoring or a right parotidectomy and selective neck dissection followed by radiation therapy. It was emphasized that monitoring was not ideal as recurrence rate is very high and will drastically decrease survival; however, the patient was lost to follow-up.

\section{Discussion}

Lymphoepithelial carcinoma (LEC) is a rare malignancy that has a strong ethnic predisposition for Eskimos in the northern circumpolar region, Mongolians, and South-east Asians..$^{1-8}$ The mean onset is 40years old, and women are more frequently affected than men regardless of ethnicity. ${ }^{2,4}$ For the populations described, this

malignancy is often found in the nasopharynx and major salivary glands and is strongly associated with Epstein-Barr virus (EBV). ${ }^{7}$ Of the salivary gland cases, $80 \%$ are found in the parotid glands, $13 \%$ in the submandibular glands, and 5\% in the minor glands. Interestingly, LEC is the most common salivary gland malignancy amongst the Inuit population and represent over $90 \%$ of all cases. . $^{1,3,6,8}$ LEC is, however, exceedingly rare in Caucasians of European ancestry and LEC in this population is often not associated with EBV.

For LEC in the head and neck, most patients develop an expanding painless mass with variable facial nerve involvement. The pathology is frequently described as a poorly differentiated carcinoma that consists of sheets of large atypical epithelial cells intermingled with benign inflammatory infiltrates rich in lymphocytes and plasma cells (lymphoid stroma) (Figure $1 \& 2$ ). The exact pathogenesis of LEC is still unclear, but its association with EBV in genetically predisposed populations suggests a role in carcinogenesis. The prognosis is usually poor and recurrence is frequent. This cancer is radiosensitive and is often treated with radiation therapy only or a combination of surgical resection, neck dissection, and radiation therapy. There are, however, no long-term follow-up studies demonstrating what combination is best to treat this cancer. ${ }^{2,6,9}$

The pathophysiology of LEC still remains unclear. It has been proposed that two-thirds arise de novo while the other one-third arises from benign lymphoepithelial lesions. ${ }^{1,2}$ The role of EBV in the pathogenesis of LEC is also puzzling. Leung et al. observed an increased LMP-1 expression in malignant epithelial cells of the salivary glands in the Hong Kong Chinese population ${ }^{7}$ and pointed out that EBV may play a role in carcinogenesis by stimulating LMP-1 production, which immortalizes malignant epithelial cells of salivary gland. LMP-1 expression, however, is less consistent in LEC of the nasopharynx. ${ }^{7}$ Also, EBV has not been associated with all LELCs such that many non-foregut-derived lymphoepithelial-like carcinomas (LELC) are usually EBV negative $e^{1,2,7,10}$ compared to foregut derived LELCs. This observation further confounds the picture and as a result, 
there is speculation that EBV may have organ specificity in genetically predisposed patients. ${ }^{1,2,6,10}$

This case exhibits some unique aspects and highlights the unclear nature of LEC. Unfortunately, lack of long-term follow-up for this patient and patients in the literature prevent us from developing a better understanding about treatment outcomes. More long-term studies need to be conducted to further elucidate the pathogenesis of LEC and the survival rate for various combination treatment modalities.

\section{Acknowledgments}

None.

\section{Conflicts of interest}

Author declares there are no conflicts of interest.

\section{Funding}

None.

\section{References}

1. Holz Sapra E, Glanzman C, Schmid S, et al. Lymphoepithelial carcinoma of the salivary glands: case reports of a rare cancer and review of the literature. Journal of Medical Research and Development. 2014;3(4):184-192

2. Ambrosio MR, Mastrogiulio MG, Barone A, et al. Lymphoepitheliallike carcinoma of the parotid gland: a case report and a brief review of the western literature. Diagn Pathol. 2013; 8:115.
3. Friborg J, Hamilton Therkidsen M, Homoe P, et al. A spectrum of Basaloid Morphology in a subset of EBV-associated lymphoepithelial carcinomas of major salivary glands. Head and Neck Pathol. 2012;6(4):445-450.

4. Schneider M, Rizzardi C. Lymphoepithelial carcinoma of the parotid glands and its relationship with benign lymphoepithelial lesions. Arch Pathol Lab Med. 2008;132(2):278-282.

5. Saku T, Cheng J, Jen KY, et al. Epstein-Barr virus infected lymphoepithelial carcinomas of the salivary gland in the RussiaAsia area: a clinicopathologic study of 160 cases. Arkh Patol. $2003 ; 65(2): 35-39$

6. Dubey P, Ha CS, Ang KK, et al. Nonnasopharyngeal lymphoepithelioma of the head and neck. Cancer. 1998;82(8):1556-1562.

7. Leung SY, Chung LP, Yuen ST, et al. Lymphoepithelial carcinoma of the salivary gland: in situ detection of Epstein-Barr virus. J Clin Pathol. 1995;48(11):1022-1027.

8. Hamilton-Dutoit SJ, Therkildsen MH, Nielsen NH. Undifferentiated carcinoma of the salivary gland in Greenlandic Eskimos: demonstration of Epstein-Barr virus DN by in situ hybridization. Hum Pathol. 1991;22:811-815.

9. Tang CG, Schmidtknecht TM, Tang GY, et al. Lymphoepithelial carcinoma: a case of a rare parotid gland tumor. Perm J.2012;16(3):60-62.

10. Jang SJ, Paik SS, Lee WM, et al. Lymphoepithelial carcinoma of the submandibular gland. J Korean Med Sci. 1997;12(3):252-255. 